\title{
Panorama da formação em psicologia para transtorno do espectro do autismo em Minas Gerais
}

\section{Overview of psychology training for autism spectrum disorde in Minas Gerais \\ Panorama de la formación en psicología para transtorno del espectro del autismo en Minas Gerais}

\section{Jardel Sander da Silva}

Professor doutor na Universidade Federal de Minas Gerais, Belo Horizonte, Brasil.

jardelss@gmail.com

ORCID - https://orcid.org/0000-0003-4785-7047

\section{Cassia Beatriz Batista}

Professora doutora na Universidade Federal de São João Del-Rei, São João Del-Rei, Minas Gerais, Brasil. cassiabeatrizb@gmail.com

ORCID - https://orcid.org/0000-0002-9393-0340

\section{Maria Luísa Magalhães Nogueira}

Professora doutora na Universidade Federal de Minas Gerais, Belo Horizonte, Brasil.

malu.mnogueira@gmail.com

ORCID - https://orcid.org/0000-0001-9024-4671

Jade Chartone Eustáquio

Graduanda na Universidade Federal de São João Del-Rei, São João Del-Rei, Minas Gerais, Brasil.

jadechartone@gmail.com

ORCID - https://orcid.org/0000-0002-1779-3751

\section{Nicole Oliveira Carvalho}

Graduanda na Universidade Federal de São João Del-Rei, São João Del-Rei, Minas Gerais, Brasil. nicoleocarvalho.93@gmail.com

ORCID - https://orcid.org/0000-0003-2715-6868

Recebido em 5 de maio 2019

Aprovado em 15 de janeiro de 2020

Publicado em 9 de março de 2020

\section{RESUMO}

Segundo os dados do relatório norte-americano (BAIO et al., 2018) a prevalência do Transtorno do Espectro do Autismo/TEA é de 1 para 59, tratando-se, portanto, de questão emergente em saúde pública. $O$ presente texto teve como objetivo desenvolver um panorama atual da formação de psicólogos quanto ao TEA. Para isso, analisamos os currículos de cursos de psicologia do estado de Minas Gerais/Brasil. Os documentos examinados foram coletados via e-mail, por meio de sites institucionais e pelas redes sociais em 2016. As disciplinas analisadas foram agrupadas em cinco grandes áreas da psicologia: educação e educação inclusiva, desenvolvimento humano, avaliação psicológica, psicopatologia e análise comportamental. De um total de 44 instituições que disponibilizaram suas matrizes curriculares para a pesquisa, foram apontadas 530 disciplinas com potencial para abordagem da temática. Como conclusão, o estudo oferece elementos que ratificam a fragilidade presente na 
http://dx.doi.org/10.5902/1984686X40092

formação em psicologia em relação ao autismo, assunto de demanda crescente na conjuntura social e de saúde brasileira.

Palavras-chave: Psicologia; Currículo; Transtorno Autístico.

\section{ABSTRACT}

About 1 in 59 children has been identified with autism spectrum disorder (ASD) according to estimates from CDC's report in 2018 (BAIO et al., 2018), which suggest that it is an issue in public health. The present paper aimed the presentantion of a current panorama on psychologists' formal education regarding the ASD. Thus, we analyze the curricula of psychology courses in the state of Minas Gerais/Brazil. The documents examined were collected via e-mail, institutional websites, and social networks in 2016. We grouped the disciplines into the 5 major areas of psychology: inclusive education and education, human development, psychological assessment, psychopathology, and behavioral analysis. In a group of 44 institutions that made their curricular matrices available, there were 530 disciplines with potential to approach the subject. This study offers elements that confirm a fragility on formal education in psychology to approach a problem that faces an increasing demand in the social and health contexts.

Keywords: Psychology, Curriculum, Autism Disorder.

\section{RESUMEN}

Según los datos del informe norteamericano (BAIO et al., 2018), la prevalencia del trastorno del espectro del autismo / TEA es de 1 a 59, tratándose, por lo tanto, de una cuestión emergente en salud pública. El presente texto tuvo como objetivo desarrollar un panorama actual de la formación de psicólogos en cuanto al TEA. Para ello, analizamos los currículos de cursos de psicología del estado de Minas Gerais / Brasil. Los documentos examinados fueron recolectados vía e-mail, sitios institucionales y redes sociales en 2016. Las disciplinas fueron agrupadas en 5 grandes áreas de la psicología: educación y educación inclusiva, desarrollo humano, evaluación psicológica, psicopatología y análisis conductual. Del total de 44 instituciones que disponibilizaron sus matrices curriculares, fueron apuntadas 530 disciplinas con potencial para abordaje de la temática. La investigación ofrece elementos que ratifican la fragilidad de la formación en psicología para un tema de demanda creciente en la coyuntura social y de salud brasileña.

Palabras clave: Psicologia, Curriculum, Transtorno Autístico.

\section{Introdução}

O autismo foi identificado como um grupo clínico pela primeira vez em 1943 por Leo Kanner. O termo autismo está radicado no grego "autos", indicando um auto-centramento. Foi usado pela primeira vez em 1910, por Bleuer1, relacionando um grupo de sintomas à esquizofrenia. Por muito tempo foi considerado um transtorno invasivo ou global do desenvolvimento, e há ainda um segmento do campo da Psicologia que insiste em manter a relação com a psicose. No entanto, pesquisas atualizadas e o DSM-V (2013) entendem o quadro como um transtorno do neurodesenvolvimento com diferentes níveis de 
http://dx.doi.org/10.5902/1984686X40092

gravidade, por isso propõem a imagem de um espectro, denominado Transtorno do Espectro do Autismo (TEA), cujo centro deficitário concentra dificuldades sociais e de comunicação Trata-se de um transtorno que indica déficits e desvios de interação e comunicação social, além de padrões de comportamento e interesses restritos e estereotipados, bem como desordens de processamento sensorial (BARANECK; et al., 2013). Os métodos diagnósticos e a própria compreensão do quadro vêm se alterando consideravelmente desde suas primeiras descrições, e felizmente vêm recebendo mais atenção científica, especialmente na última década.

A leitura do autismo como um espectro relaciona-se à percepção de estudiosos (ASPERGER, 1994; WING, 1997) que, desde a conceituação do transtorno, observaram uma plasticidade importante no quadro, em termos de gradação, distúrbios e etiologias. Trabalharemos na perspectiva de que o TEA é um transtorno do neurodesenvolvimento que combina fatores genéticos e ambientais ainda não bem definidos, em que intervenções e tratamentos com reconhecida comprovação científica podem ser altamente benéficos, favorecendo a autonomia desses indivíduos na vida adulta. Além disso, é necessária uma perspectiva social para seu entendimento: "A compreensão do autismo só será obtida por meio de um exame mais aprofundado tanto através das culturas quanto dentro delas"' (GRINKER; DALEY; MANDELL, 2013, p. 830). Os autores argumentam que diferenças culturais vêm a influenciar a epidemiologia, o diagnóstico e o tratamento do TEA no mundo.

Os dados oficiais sobre a prevalência do autismo no Brasil são frágeis. Foi realizada em 2011 uma pesquisa piloto no município de Atibaia (São Paulo) cujo resultado indicou a taxa de 1 a cada 370 crianças (PAULA; BELISÁRIO FILHO; TEIXEIRA, 2011). O relatório norte-americano do Centers for Disease Controland Prevention (CDC), de 2018, indica o índice de prevalência do TEA sendo de 1 para 59 indivíduos. Percebe-se um aumento notável da prevalência chegando a taxas de aumento de $80 \%$, desde 2008, ainda que os números possam variar bastante (BAIO, 2012). A organização não-governamental brasileira Autismo e Realidade estima uma população de 1,9 milhão de pessoas com transtorno do espectro do autismo (TEA) no país. Trata-se, portanto, de uma questão emergente de saúde pública que já vem sendo anunciada dessa forma em outros países, atingindo números expressivos de pessoas de diversas camadas sociais e distintas origens culturais. Não temos estudos comparativos sólidos, mas já é possível antever que a forma de compreender e conceituar o que é o TEA impacta os recursos que 
http://dx.doi.org/10.5902/1984686X40092

profissionais e famílias têm disponíveis para a intervenção adequada, o que, por sua vez, impacta o prognóstico.

Um elemento consensual dentro da temática do autismo é a importância da intervenção precoce; no entanto, as estatísticas brasileiras indicam atraso considerável nessa tarefa. O estudo de Ribeiro e colaboradores (2017) indica que o diagnóstico só é estabelecido próximo dos 6 anos, ainda que as mães tenham manifestado suas preocupações iniciais, geralmente com o pediatra, por volta dos 23 meses. Na ausência de capacitação mais aprofundada pelos profissionais de saúde, muitos casos deixam de ser diagnosticados ou o recebem tardiamente. Tal quadro pode comprometer 0 prognóstico, aumentando os custos, tanto para a família, quanto para o Estado, já que aqueles que não receberam estimulação adequada, preferencialmente na primeira infância, geralmente apresentam um quadro, quando adultos, de evidente dependência social e econômica (ROGERS; VISMARA, 2008; CIDAV et al., 2017; KLIN, 2006).

Ainda não foram identificados marcadores biomédicos, o que torna o diagnóstico puramente clínico, pautado em fatores indicadores do TEA na percepção de psicólogos, psiquiatras e neurologistas. Sabe-se que o apontamento para o diagnóstico ou, pelo menos, para o risco de autismo precisa ser feito o quanto antes, de modo a viabilizar a intervenção precoce que torna o prognóstico mais favorável, como indica a literatura especializada. A intervenção precoce atua preventivamente, evitando que se instale uma cascata de prejuízos em virtude dos desafios sociocomunicativos presentes no TEA, tais como baixo contato visual, imitação falha, pouca ou nenhuma atenção compartilhada, ou seja, a fragilidade no interesse pelo outro gera uma constante perda de oportunidades de aprendizagem que vai se agravando e gerando a cronificação dos sintomas (BAGAIOLO et al., 2018; PINTO et al., 2016; COSTA, 2014; ROGERS; DAWSON, 2014; DAWSON, 2008). A Sociedade Brasileira de Pediatria publicou dois documentos científicos sobre TEA, um específico sobre rastreamento (ARAÚJO; LEYSER, 2017) e outro que orienta amplamente sobre o TEA (ARAÚJO et al., 2018), o que indica o aumento da atenção dada a essa condição no contexto brasileiro.

Em 2001, Shah (2001) conduziu um estudo na Inglaterra, no qual entrevistou 250 estudantes em diferentes períodos da graduação em Medicina. A pesquisa comparou as respostas dos graduandos do $1^{\circ}$ e do $4^{\circ}$ ano quanto a questões relativas ao TEA, tais como diagnóstico, cura, sintomatologia e prognóstico, identificando que os alunos de fim de curso apresentaram respostas melhores tanto sobre critérios diagnósticos quanto em 
http://dx.doi.org/10.5902/1984686X40092

relação aos sintomas centrais do quadro, mas não sobre possíveis causas, perfis de QI, prognóstico e tratamento. Assim, o estudo conclui que uma ênfase maior no TEA se faz necessária na graduação em saúde. Como já se passaram mais de 15 anos desde a realização dessa pesquisa, é inevitável nos questionarmos: será que estamos caminhando na direção de produzir uma formação de profissionais da área de saúde atenta à necessidade de ênfase em TEA?

Paula e colaboradores (2016), em um estudo recente realizado em universidades da cidade de São Paulo, buscaram avaliar o nível de conhecimento a respeito do TEA em estudantes do $9^{\circ}$ e $10^{\circ}$ período do curso de psicologia. A amostra avaliada apresentou um nível de conhecimento dos sujeitos de regular a baixo, no que tange aos aspectos referentes ao quadro clínico, etiologia e dados epidemiológicos.

Enquanto o TEA toma a atual dimensão de um problema emergente de caráter em saúde pública, o desenvolvimento de estudos e a promoção de debates que reorientam de forma geral a formação em saúde avançam e ganham cada vez mais força na literatura científica nacional (FEUERWERKER; ALMEIDA, 2003; CECCIM; CARVALHO, 2006; BATISTA, 2013). Dessa forma, pesquisar a formação profissional e acadêmica de sujeitos e sua forma de agir e pensar no campo da saúde tornam-se desafios permanentes, no sentido de que tal formação deve ser sintonizada com a realidade social, em relação a novos problemas, a diagnósticos, a tratamentos, a redes de assistência e reabilitação. Esse fato é especialmente relevante quando nos referimos ao TEA, posto que se trata de um quadro imerso ainda em um oceano de controvérsias, alvo de produções distintas de significado (ORTEGA, 2013).

Pesquisar a formação de profissionais em saúde, especificamente de psicólogos, nos remete primeiramente, à graduação e, consequentemente, aos estudos de currículos que assumem uma posição estratégica, uma vez que estes documentos inserem-se numa perspectiva de construção social e política (PACHECO, 2002), alicerçada na diversidade e pluralismo, mas também na manifestação de processos e práticas de poder. Assim, tomaremos o currículo como discurso social e, sobretudo, como documento de identidade (SILVA, 2003), capaz de transcender aos aspectos teóricos e técnicos envolvidos na formação profissional, isso é, capaz de representar relações culturais, disputas de concepções e de interesses na prática educativa. Assim, produzir um currículo é assumir um compromisso na formação de sujeitos que cumpram as demandas da sociedade em seus diferentes espaços e tempos sociais. 
http://dx.doi.org/10.5902/1984686X40092

É possível identificar um crescimento da preocupação com a formação de profissionais de saúde e de educação em todo o mundo, que tem buscado direcionar a assistência para a concepção ampliada de saúde, pautada em sua prevenção e promoção e no entendimento do ser humano em suas dimensões biopsicossociais e único em suas vicissitudes. O presente trabalho busca refletir - a partir de um recorte específico - se, atualmente, nossos sistemas educacionais preparam profissionais para atuar num contexto de atenção ao TEA na dimensão de saúde pública, tanto nos aspectos de ação junto à construção de políticas públicas, quanto no confronto aos ainda vigentes modelos sociais nos quais não há espaço para a diferença.

\section{Percurso metodológico}

Trata-se de uma pesquisa de cunho qualitativo cujo objetivo traduz-se em compreender o panorama da formação de psicólogos para a identificação e manejo do TEA nos cursos de graduação em psicologia de universidades do estado de Minas Gerais.

$\mathrm{Na}$ aproximação da formação em psicologia, a análise documental (BORRIONE E CHAVES, 2004; CELLARD, 2008) foi ferramenta metodológica central que possibilitou a coleta, sistematização e análise de informações produzidas por certas instituições de ensino em diálogo com as normativas ministeriais e também com seus destinatários e suas intencionalidades envolvidas nas propostas formativas.

Para cumprir este fim, foi utilizada a análise documental curricular, projetos pedagógicos de cursos, matrizes e planos de ensino, que além de aspectos estruturais e técnicos da formação, aludem às concepções defendidas no processo educativo.

$\mathrm{Na}$ análise das fontes documentais, um enfoque temático foi adotado (BARDIN, 2004) e construído a partir das referências teóricas de estudos anteriores das autoras sobre formação em saúde no curso de psicologia (CARMONA e BATISTA, 2016; BATISTA, CARMONA E FONSECA, 2014) e mais especificamente pelos estudos sobre TEA do grupo de pesquisa do Laboratório de Estudo e Extensão em Autismo e Desenvolvimento da UFMG (BORGES; NOGUEIRA, 2018)

Existem cerca de 60 instituições que oferecem regularmente graduação em psicologia no estado de Minas Gerais (INEP, 2017), sendo que mais de $85 \%$ são cursos privados. Foram identificados 57 cursos, sendo seis de universidades públicas, e 51 de instituições privadas. Nota-se uma evidente concentração de oferta na região 
http://dx.doi.org/10.5902/1984686X40092

Metropolitana de Belo Horizonte. Há instituições com mais de um curso, sendo distribuídos por diferentes regiões, alguns com currículos idênticos, outros não, sendo que diferentes polos vinculados à mesma rede de ensino, utilizam o mesmo currículo.

As fontes de informações foram extraídas de páginas oficiais das instituições, bem como de e-mails enviados para coordenadorias e gestores dos cursos, no período de abril a junho de 2016. A fim de construir um painel geral e simultaneamente representativo, optamos pela análise de apenas uma versão de cada matriz, de forma que, ao final, foram estudadas 44 matrizes curriculares ${ }^{1}$ distintas, ou seja, pertencentes a 44 cursos de psicologia. Este documento permitiu identificar disciplinas que abordam ou apresentam potencialidades de abarcar a temática do TEA nos cursos de psicologia.

Foram identificados 57 cursos, sendo seis de universidades públicas, e 51 de instituições privadas. Nota-se uma evidente concentração de oferta na região Metropolitana de Belo Horizonte. Há instituições com mais de um curso situados em diferentes regiões, alguns com currículos idênticos, outras não, sendo que diferentes polos vinculados à mesma rede de ensino, utilizam o mesmo currículo.

Para compreensão do panorama da formação sobre o TEA nas graduações mineiras de psicologia, dividimos a análise de documentos em dois momentos. No primeiro momento, foi elaborada uma tabela contendo: nome da instituição de ensino superior, o regime de administração (pública ou privada), o nome da disciplina com potencial para abordagem da temática do TEA, a carga horária, o período, e por fim, o tipo de oferecimento da disciplina (optativa ou obrigatória).

Todos os 44 cursos foram contactados por e-mail institucional para obter o Projeto Pedagógico de Curso atualizado, mas apenas três responderam. Assim, a busca se estendeu até fevereiro de 2017 com a incorporação do uso das redes sociais, para ampliar nosso acesso, inclusive por meio de contato também com os alunos. Os 7 Projetos Pedagógico de Curso coletados pela equipe são de cursos em que os pesquisadores atuam, atuaram ou têm colegas que disponibilizaram os documentos, totalizando a coleta de 15 documentos compostos por 10 Projetos Pedagógico de Curso, 2 Planos de Ensino ${ }^{3}$ e 3 ementários. Destes 15 documentos que trazem informações mais detalhadas com proposta pedagógica, descrição de conteúdos e de referenciais teóricos, quatro são de instituições públicas. Cabe esclarecer que os documentos de fácil acesso público foram oriundos, em sua maior parte, de instituições federais, o que não deixa de ser um dado importante e alarmante sobre o acesso à informação no âmbito privado. 
http://dx.doi.org/10.5902/1984686X40092

As análises dos Projetos Pedagógicos de Curso foram realizadas no segundo momento, que contou com grande dificuldade de acesso aos documentos e precariedade das informações, ora incompletas, ora desatualizadas. Essa dificuldade apresentou-se como um contexto desafiador para o cumprimento dos objetivos propostos neste estudo.

Em resumo, a coleta de dados foi feita em dois momentos e usando recursos distintos. No primeiro momento, identificamos os documentos grades curriculares disponibilizadas livremente, por meio de busca nos sites. Na segunda etapa, acionamento da rede, seja por e-mail, telefone ou até redes sociais para obter os projetos pedagógicos. Desse modo, dos 57 cursos de Psicologia, encontrados em Minas Gerais, resgatamos, por meio dos sites, inicialmente, 44 matrizes curriculares ou grades. A partir desse universo, coletamos 15 documentos com informações substanciais, sendo 4 materiais oriundos de instituições públicas e 11 de instituições privadas.

Embora os 15 documentos sejam um contingente numericamente pouco representativo - se comparado ao total de instituições que oferecem graduação em psicologia -, foram todos analisados criteriosamente a fim de se produzir uma visão inicial acerca da abordagem do TEA na proposta político-pedagógica dos cursos.

No processo de sistematização dos dados coletados, foram selecionadas e categorizadas disciplinas que poderiam abordar conteúdos teórico-metodológicos do campo do TEA, ou seja, aquelas que continham em suas ementas os termos educação, desenvolvimento, psicopatologia, avaliação psicológica e comportamento. Classificamos tais disciplinas entre 5 grandes áreas da psicologia, de acordo com a perspectiva epistemológica de abordagem, a saber: Educação e Educação Inclusiva, Desenvolvimento Humano, Psicopatologia, Avaliação Psicológica e Análise Comportamental.

$\mathrm{Na}$ área da Educação, encontram-se disciplinas voltadas ao contexto escolar e da psicopedagogia, bem como à formação do psicólogo para o atendimento específico de pessoas com deficiência; nessa última área, a denominação anterior era para atendimento aos portadores de necessidades especiais, e que hoje também é denominada como público alvo da educação especial. Reconhecemos as dificuldades que envolvem a nomenclatura dessa área, atravessada por diversas especificidades. Deste modo, incluímos em nossas buscas as diversas perspectivas, contidas nessa polifonia. 
http://dx.doi.org/10.5902/1984686X40092

$\mathrm{Na}$ área do Desenvolvimento, foram alocadas as disciplinas obrigatórias de Psicologia do Desenvolvimento, bem como disciplinas que contemplam perspectivas da neuropsicologia e neurociência, de forma geral.

Consideramos interessante estabelecer uma área específica para Análise Aplicada do Comportamento, uma vez que há vasta literatura que indica sua eficácia na intervenção no TEA. , tendo sido confundida com um modelo específico de tratamento para o transtorno. Uma vez que encontramos expressivo material sobre TEA desenvolvido a partir desse viés e que o campo da Psicopatologia já estava devidamente contemplado, buscamos assim, ter acesso às abordagens para TEA em mais de uma perspectiva.

Do mesmo modo, a área da Avaliação Psicológica foi recortada, pois poderia indicar a capacitação para o diagnóstico do transtorno, por meio de instrumentos de avaliação desenvolvidos especificamente para TEA, assim como disciplinas que abordem os aspectos do neurodesenvolvimento referentes ao transtorno.

$\mathrm{Na}$ área da Psicopatologia tanto numa perspectiva mais convencional da nosologia; quanto em perspectivas mais críticas encampadas pelo movimento antimanicomial, está o campo da saúde mental. Aqui também está a psicopatologia da infância, geralmente sustentado por leituras psicanalíticas.

A divisão em grandes áreas possibilita uma percepção sobre a pluralidade discursiva que permeia a formação em saúde sobre esse transtorno, permitindo, ainda, o apontamento de autores-pista do TEA que trabalham com a perspectiva teórica característica de cada uma das áreas. A equipe elencou 43 autores de referência que foram, então, empregados como indicadores ou autores-pista, no levantamento do material. Estes 43 autores-pista foram selecionados por serem aqueles com efetiva produção na área, ou seja, que apresentavam pelo menos 2 publicações sobre TEA, nacionais ou internacionais.

Com isso, buscamos a pluralidade discursiva que configura a formação em psicologia sobre a temática do TEA, visando avançar na compreensão dos processos formativos.

\section{Apresentação e análise de dados}

As iniciativas de mudanças na formação em saúde são ratificadas em documentos como o as Diretrizes Curriculares Nacionais (DCN). No caso específico dos currículos de psicologia, a resolução mais recente - Resolução CNE/CES No5 de março de 2011 
http://dx.doi.org/10.5902/1984686X40092

(BRASIL, 2011) - estabelece o compromisso com a educação que reconheça a diversidade de perspectivas necessárias para a compreensão do ser humano em sua complexidade e multideterminação de fenômenos psicológicos. Discorre-se ainda sobre a na exigência de um projeto garantidor de habilidades e competências que propicie a compreensão ampla e integral dos processos psicológicos tanto individuais quanto coletivos.

Art. 8ㅇ As competências reportam-se a desempenhos e atuações requeridas do formado em Psicologia, e devem garantir ao profissional o domínio básico de conhecimentos psicológicos e a capacidade de utilizá-los em diferentes contextos que demandam a investigação, análise, avaliação, prevenção e atuação em processos psicológicos e psicossociais e na promoção da qualidade de vida. Dentre as competências destacam-se:

I - analisar o campo de atuação profissional e desafios contemporâneos;

[...] III - identificar e analisar necessidades de natureza psicológica, diagnosticar, elaborar projetos, planejar e agir de forma coerente com referenciais teóricos e características da população-alvo;

[...] VI - avaliar fenômenos humanos de ordem cognitiva, comportamental e afetiva, em diferentes contextos;

VII - realizar diagnóstico e avaliação de processos psicológicos de indivíduos, de grupos e de organizações;

VIII - coordenar e manejar processos grupais, considerando as diferenças individuais e socioculturais dos seus membros. (BRASIL, 2013)

Ainda que não pontue especificamente sobre a assistência aos indivíduos com TEA, as DCN da psicologia apontam para uma formação voltada às demandas sociais atuais e/ou potenciais que se apresentarem. Isto é, embora não haja referências diretas, as DCN orientam para a possibilidade de uma capacitação profissional que garanta habilidades de manejo e desenvolvimento de políticas direcionadas ao TEA.

Tratando ainda das DCN, um dos objetivos a serem contemplados pelo projeto pedagógico complementar, no que concerne à formação de professores de psicologia, é o de possibilitar uma formação de profissionais comprometidos com transformações político-sociais, de forma a adaptar sua prática pedagógica a uma educação inclusiva. Essa proposta pretende fortalecer a construção de políticas públicas de educação, bem como formar professores capazes de refletir criticamente sobre o processo de ensinoaprendizagem para os diferentes sujeitos em suas particularidades. Pode-se dizer, portanto, que a prática desse profissional é um importante disparador de questionamentos às políticas educacionais, aos projetos e às ações pedagógicas, tornando-se também, um 
http://dx.doi.org/10.5902/1984686X40092

núcleo dentro das DCN capaz de trabalhar políticas, ações e competências voltadas à assistência de pessoas com TEA.

Coadunando-se a esse contexto, a Nota Técnica № 24/2013/MEC/SECADI/DPEE (BRASIL, 2013) orienta sistemas de ensino em geral para a implementação da Política Nacional de Proteção dos Direitos da Pessoa com TEA. Dentre as diretrizes que tratam da efetivação do direito à educação para as pessoas com TEA, destacamos: a transversalidade da educação especial desde a educação infantil até a educação superior; o Atendimento Educacional Especializado; a formação de professores para o Atendimento Educacional Especializado (AEE), e demais profissionais da educação para a inclusão escolar; e articulação intersetorial na implementação das políticas públicas.

\begin{abstract}
A intersetorialidade na gestão das políticas públicas é fundamental para a consecução da inclusão escolar, considerando a interface entre as diferentes áreas na formulação e na implementação das ações de educação, saúde, assistência, direitos humanos, transportes, trabalho, entre outras, a serem disponibilizadas às pessoas com transtorno do espectro autista. [...] Além disso, a formação dos profissionais da educação possibilitará a construção de conhecimento para práticas educacionais que propiciem o desenvolvimento sócio-cognitivo dos estudantes com transtorno do espectro autista. (BRASIL, 2013)
\end{abstract}

Embora a Nota Técnica se debruce sobre aspectos organizacionais e estruturais que asseguram a plena inserção educacional de pessoas com o TEA, o reconhecimento do direito à inclusão desses sujeitos nos diversos ambientes sociais, juntamente com a promoção de condições que garantam seu desenvolvimento pessoal e social, permitemnos considerar que os serviços de assistência, nesse caso, especificamente os serviços educacionais, têm recebido atenção jurídica que reconhece e aponta para a necessidade da reestruturação adequada, de modo a atender as demandas singulares dessa população. Isso pode ser indicado pela institucionalização do chamado AEE Atendimento Educacional Especializado -, um conjunto de serviços e atividades oferecidos complementarmente à escolarização. Em tese, este deveria eliminar as barreiras do processo educativo a partir da flexibilização da assistência pedagógica Resolução CNE/CEB n 4/2009 - (BRASIL, 2009). No entanto, pesquisas indicam que sua presença ainda não faz parte da realidade dos serviços educacionais oferecidos pela rede pública (GOMES; MENDES, 2010), sendo ainda mais raros no que tange à rede privada, segundo relatos das famílias de pessoas com TEA (NOGUEIRA; VICARI; BRUN, 2016). Além disso, percebe-se que a relação entre saúde e educação fica tensionada, uma vez que as famílias dependem dos laudos médicos para acessarem os direitos, 
http://dx.doi.org/10.5902/1984686X40092

como o acesso ao AEE e a um profissional de apoio à inclusão, o mediador, mas enfrentam dificuldades diversas para conseguirem esses documentos. A revisão realizada por Nunes, Azevedo e Schmidt (2013) sugere que há ainda um desconhecimento sobre TEA, no âmbito da educação, bem como a carência de estratégias pedagógicas específicas, impactando negativamente as oportunidades de aprendizado nas crianças com TEA nas escolas brasileiras. O aumento da presença de estudantes com TEA nas escolas não implicou, efetivamente, na presença de recursos e metodologias adequadas às especificidades do desenvolvimento no TEA. Assim, o incentivo para a formação de profissionais especializados e devidamente capacitados para o atendimento à pessoa com TEA segue como preocupação dos órgãos governamentais.

[...] O Plano de AEE do estudante com transtorno do espectro autista contempla: a identificação das habilidades e necessidades educacionais específicas; a definição e a organização das estratégias, serviços e recursos pedagógicos e de acessibilidade; o tipo de atendimento conforme as necessidades de cada estudante; o cronograma do atendimento e a carga horária, individual ou em pequenos grupos. [...]

[...] Este atendimento prevê a criação de redes intersetoriais de apoio à inclusão escolar, envolvendo a participação da família, das áreas da educação, saúde, assistência social, dentre outras, para a formação dos profissionais da escola, o acesso a serviços e recursos específicos, bem como para a inserção profissional dos estudantes. A modalidade da educação especial disponibiliza o atendimento educacional. [...] (BRASIL, 2013)

Nessa perspectiva, e sabendo que o exercício da cidadania e a plena integração social da pessoa com TEA dependem de uma rede de esforços intersetoriais, que sobrepujem o campo educacional, isto é, que envolvam áreas como saúde e assistência social, todas elas escopo de atuação para profissionais psicólogos, novamente direcionamo-nos para a necessidade de compreender as possíveis lacunas em relação à abordagem do autismo no currículo da referida profissão, objetivo desse trabalho.

Foram contabilizados 530 registros de disciplinas capazes de contemplar a temática do TEA, agrupadas nas grandes áreas acima mencionadas, para as quais foram identificados os seguintes números: o campo do Desenvolvimento Humano contou com 133 registros (ou seja, títulos de disciplinas); o da Educação com 123 registros seguido da Psicopatologia, com 114 registros; o da Análise Comportamental, com 85 registros e logo depois, o da Avaliação Psicológica, com 80 registros. Os dados tornam-se mais claros por meio da representação gráfica a seguir (Gráfico 1). 
http://dx.doi.org/10.5902/1984686X40092

Gráfico 1: Distribuição do número de registros segundo as áreas do Desenvolvimento, Psicopatologia, Educação, Análise Comportamental e Avaliação Psicológica

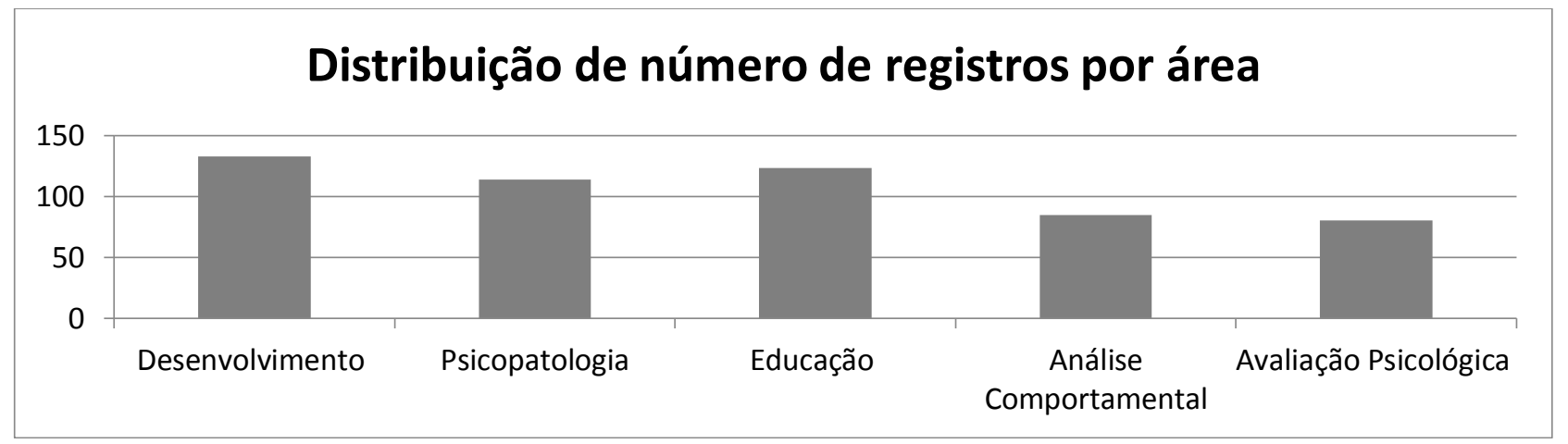

Fonte: Dados da pesquisa, 2019.

Os dados foram avaliados e categorizados por meio de uma escala numérica de zero a quatro, sendo zero nenhuma menção aos autores pista ou literatura afim e quatro a abordagem direta ao tema com bibliografia atualizada, referente a publicações após 2006 e até 2017.

Observamos o predomínio de disciplinas das áreas de Desenvolvimento Humano, Educação e Psicopatologianas matrizes curriculares dos cursos de psicologia elencados. Observamos que $80 \%$ das disciplinas de Desenvolvimento e de Psicopatologia são ofertadas na forma obrigatória, sendo as demais de caráter optativo.

Atualmente, o TEA é considerado um transtorno do desenvolvimento, que envolve comprometimento importante de habilidades sociocomunicativas. O conhecimento acerca do desenvolvimento humano, sobretudo o desenvolvimento infantil em seus aspectos psíquicos, físico-motores e afetivo-sociais permite uma intervenção precoce, e garante a possiblidade de mudança no quadro e de uma melhor evolução da criança.

Tanto o campo da Educação quanto da Psicopatologia, contempla $22 \%$ das disciplinas identificadas. Tomando como base documentos de referência para a graduação, como a Nota Técnica do MEC avaliada anteriormente, bem como as DCN nas disposições sobre a formação de professores em psicologia, é importante que tal porcentagem possa garantir habilidades e competências profissionais para a produção de atendimento educacional especializado e inclusivo. A boa formação de psicólogos e outros profissionais da área educacional possibilitam o aprimoramento de artifícios pedagógicos capazes de construir processos de significação da experiência escolar e com isso assegurar o desenvolvimento sociocognitivo do estudante com TEA. 
http://dx.doi.org/10.5902/1984686X40092

As reflexões elaboradas sobre as matrizes curriculares podem ser aprofundadas através do estudo criterioso dos Projetos Pedagógicos de Curso e Planos de Ensino, constituindo-se no segundo momento da nossa análise de dados. Dividimos então, os documentos quanto à presença ou não de referências às palavras chave: 'autismo', 'transtorno autista', 'transtorno do espectro autista', 'transtorno autístico' e 'TEA'; e também quanto à menção ou não aos autores-pista, conforme descrito acima, de cada grande área de enquadramento das disciplinas. É importante sublinhar que nem sempre estava claro no título da disciplina a presença da discussão sobre TEA, por isso, complementamos o critério utilizando os autores-pista. De forma geral, observamos que 5 dos documentos arquivados, sendo 3 ementários e 2 projetos político-pedagógicos, eram incompletos no quesito apresentação de referências bibliográficas, impossibilitando a investigação sobre autores-pista e, consequentemente, sobre a perspectiva mais frequentemente utilizada no embasamento dos estudos sobre autismo. Apesar disso, procuramos por referências às palavras-chave destacadas em todos os documentos, inclusive os sem referencial bibliográfico.

Constatamos que do total de 15 documentos, 10 não apresentavam referências diretas às palavras-chave destacadas. Ainda nessa perspectiva, apenas 2 arquivos apresentaram menções aos termos destacados, respectivamente 'transtorno autista', 'autismo' e 'autista', especificamente nos planos de ensino de suas disciplinas. Quanto aos 3 documentos restantes, todos apresentaram alusão ao termo 'Transtorno do Espectro do Autismo', entretanto, diferentemente do que pretendia avaliar o projeto, a abordagem discorria sobre núcleos de apoio psicopedagógico e programas de acompanhamento a acadêmicos com Transtorno Invasivo do Desenvolvimento, em especial o TEA. Ou seja, embora a temática estivesse presente na discussão políticoideológica do projeto pedagógico, ela não apareceu como forma de direcionamento da formação profissional, e sim como proposta que fazia jus às proposições da Lei de Proteção aos Direitos da Pessoa com Transtorno do Espectro do Autismo.

Um elemento que julgamos importante analisar foi o grau de atualidade das referências bibliográficas dos cursos, justamente por ser o TEA um objeto emergente do campo científico e que recebeu alterações e contribuições significativas para sua compreensão na última década. Dos 10 projetos pedagógicos encontrados, 6 retratavam as referências bibliográficas das disciplinas. Nestes 6 projetos, analisamos as disciplinas num total de 55. 
http://dx.doi.org/10.5902/1984686X40092

No quesito atualidade, o material bibliográfico de referência concentra-se entre 2000 e 2008. É preciso sublinhar que 6 disciplinas (11\%) fundamentam seus conteúdos a partir de uma literatura anterior ao ano 2000. A análise de dados indica que a maior parte do referencial teórico utilizado ignora a produção científica mais atualizada, encontrando-se, portanto, desatualizado.

Somente 1 disciplina aborda a questão a partir de um referencial teórico mais atualizado (última década), mas ainda sem indicação de literatura específica para TEA. Vale ressaltar que o DSM-V (2013), manual diagnóstico que trouxe importantes mudanças para a descrição nosológica do TEA, foi publicado no ano de 2013, mas o DSM-IV é o mais utilizado, tendo sido publicado em 1994.

Quanto aos Projetos pedagógicos dos cursos que apresentavam menção aos autores-pista, foram encontradas, ao todo, 11 referências, sendo 8 de autores do campo da educação e 3 da área de psicopatologia.

Ainda analisando os referenciais bibliográficos, deparamo-nos com uma questão importante que atravessa o cenário da produção de conhecimento e da orientação para o tratamento para o TEA: na área de Psicopatologia há uma prevalência de autores de perspectiva psicanalítica, ou seja, todos os autores-pista encontrados são dessa orientação. As premissas que direcionam o referencial teórico da psicanálise partem de um pressuposto de que o TEA é um desvio do desenvolvimento cuja etiologia se dá a partir de fatores preponderantemente afetivos, desconhecendo o autismo enquanto um transtorno do neurodesenvolvimento de base fundamentalmente biológica e orgânica. Não existem pesquisas substanciais que qualifiquem o saber que a Psicanálise produz sobre o TEA que sejam capazes de justificar a predominância dessa abordagem nos currículos, sobretudo quando comparado a outras áreas que possuem um escopo teórico mais consistente, como as produções teóricas pautadas na psicologia do desenvolvimento e na análise aplicada do comportamento (ROGERS; DAWSON, 2014). Ainda em relação à área da Psicopatologia, sendo uma disciplina obrigatória que exerce um predomínio no currículo de psicologia, cabe rediscuti-la atualizando o debate e apresentando as ressalvas pertinentes.

Mesmo diante do pequeno contingente de documentos que arquivamos, foi possível perceber que a abordagem da temática do TEA como uma disciplina específica, ou como um subtema dentro de disciplinas de cada grande área da psicologia é ainda extremamente exígua, se comparada à demanda social que esse transtorno vem 
http://dx.doi.org/10.5902/1984686X40092

assumindo nos últimos anos. Ou seja, para uma formação generalista e voltada para necessidades de saúde conforme preconiza as DNC, não encontramos o ensino geral mínimo sobre TEA na formação dos psicólogos em Minas Gerais.

Constatamos, a partir dessa análise preliminar, que existe um amplo espaço para uma possível discussão sobre o autismo: apontamos mais de 500 disciplinas com potencial para suscitar essa discussão nas matrizes curriculares. Ainda assim, a realidade dos projetos pedagógicos e planos de ensino não confirmou essa hipótese.

\section{Considerações finais}

O acesso e, sobretudo, o conhecimento sobre estruturação e organização dos currículos de graduação no ensino superior nos permitem apontar conhecimentos atuais, questionamentos, experiências, concepções e posicionamentos circulantes sobre um determinado conteúdo de interesse nos espaços de formação profissional. Inegavelmente, um dos grandes aprendizados desse trabalho é que, enquanto a contemporaneidade se caracteriza por um intenso fluxo informacional, a dimensão de acessibilidade a essas informações e às bases de dados, como um todo, é em muitos aspectos comprometida. A dificuldade de acessar documentos de caráter público, tais como os projetos políticopedagógico dos cursos de graduação em psicologia, dificultou uma análise mais aprofundada e representativa acerca do panorama da formação sobre o autismo nos cursos de graduação em psicologia de universidades do estado de Minas Gerais.

Parcerias entre núcleos de pesquisa em múltiplas cidades do estado podem ser firmadas a fim de que tais documentos possam ser requisitados pessoalmente, um recurso adicional à busca virtual que se mostrou menos produtiva do que o esperado. Além disso, no que diz respeito às universidades particulares faz-se evidente a necessidade de mais transparência e abertura para o acesso aos currículos, pois um número restrito de arquivos foi encontrado, mesmo após uma vasta busca que envolveu múltiplos meios de pesquisa: contato com coordenadores via e-mail, requisição formal e contato direto com os estudantes e professores por meio de redes sociais. Pesquisas futuras podem ser realizadas com visitas institucionais e entrevistas com coordenadores e professores de disciplinas das diversas áreas para conhecer as práticas pedagógicas oferecendo mais elementos para ampliarmos o conhecimento e o debate sobre o TEA nos cursos de saúde. 
http://dx.doi.org/10.5902/1984686X40092

Com relação ao material disponibilizado e analisado, percebe-se a notável urgência de atualizações e revisões curriculares de modo que a formação destes profissionais diante da questão do TEA possa se ancorar nos paradigmas discutidos pela literatura científica atual. Novos estudos deverão se aprofundar em perspectivas teóricas e outros cenários de aprendizagem universitária como projetos de pesquisa e extensão, eventos, grupos de estudos, pós-graduações e publicações de professores universitários. Ainda assim, a formação generalista que atinge todos os alunos no que se refere às atividades de ensino, principalmente às disciplinas obrigatórias, não contempla de forma satisfatória as demandas sociais e de saúde emergentes, como é o Transtorno do Espectro do autismo.

\section{Referências}

ARAÚJO, Liubiana A.; LEYSER, Márcio. Triagem precoce para Autismo/ Transtorno do Espectro Autista. 2017. Documento Científico da Sociedade Brasileira de Pediatria. Dispoonível em: https://www.sbp.com.br/fileadmin/user_upload/2017/04/19464bDocCient-Autismo.pdf. Acesso em: 23 abr.2018.

ARAÚJO, Liubiana A. et al. Manual de Orientação: transtorno do espectro do autismo. Sociedade Brasileira de Pediatria - Departamento de Pediatria do Desenvolvimento e Comportamento, n.5, [S.I.: s.n.], abr.2019. Disponível em:

https://www.sbp.com.br/fileadmin/user_upload/Ped._Desenvolvimento_-_21775b-MO__Transtorno_do_Espectro_do_Autismo.pdf. Acesso em: 20 set.2019.

ASPERGER, Hans. Die 'aunstisehen Psychopathen' im Kindesalter. Archiv für Psychiatrie und Nervenkrankheiten. 117, 76-136, 1994 [1944].

BAGAIOLO, Leila Felipe. et al. Capacitação parental para comunicação funcional e manejo de comportamentos disruptivos em indivíduos com Transtorno do Espectro Autista. Cadernos de Pós-Graduação em Distúrbios do Desenvolvimento, São Paulo, v.18, n.2, p.46-64, jul./dez. 2018. Disponível em:

http://editorarevistas.mackenzie.br/index.php/cpgdd/article/view/12113/7484. Acesso em: 14 jun.2019

BAIO, Jon. Prevalence of Autism Spectrum Disorders: Autism and Developmental Disabilities Monitoring Network, 14 Sites, United States, 2008. Morbidity and Mortality Weekly Report. Surveillance Summaries. Centers for Disease Control and Prevention, v.61, n.3. 2012.

BAIO, Jon et al. Prevalence of Autism Spectrum Disorder Among Children Aged 8 Years - Autism and Developmental Disabilities Monitoring Network, 11 Sites, United States, 2014, Surveillance Summaries, [S.I.: s.n.], v.67, n.6, 1-23, apr. 27 2018. Disponível em: https://www.cdc.gov/mmwr/volumes/67/ss/ss6706a1.htm. Acesso em: 15 set. 2019. 
http://dx.doi.org/10.5902/1984686X40092

BARANEK, Grace. T. et al. Hyporesponsiveness to social and nonsocial sensory stimuli in children with autism, children with developmental delays, and typically developing children. Development and Psychopathology, v.25, n. 2, 307-320, 2013.

BATISTA, Cássia Beatriz. Movimentos de reorientação da formação em saúde e as iniciativas ministeriais para as universidades. Barbarói, (38), 97-125, 2013.

BATISTA, Cássia Beatriz; CARMONA, Daniele Souza; FONSECA, Sara. Lopes. A formação em saúde e os cenários de aprendizagem no curso de psicologia. Psicologia Argumento (PUCPR. Online), v.32, p.17-25, 2014.

BARDIN, Laurence. Análise de conteúdo. 3.ed. Lisboa: Ed. 70, 2004.

BORGES, Adriana A.P.; NOGUEIRA, Maria Luísa M. (Org.). O Aluno com Autismo na Escola (série Toda criança pode aprender). 1ed. Campinas: Mercado das Letras, 2018, v. 1.

BORRIONE, Roberta; CHAVES, Antonio Marcos. Análise documental e contexto de desenvolvimento: estatutos de uma instituição de proteção à infância de Salvador, Bahia.

Estud. psicol. Campinas [online], vol.21, n.2, pp.17-27, 2004. Disponível em:

http://www.scielo.br/pdf/estpsi/v21n2/a02v21n2.pdf. Acesso em: 25 fev.2018.

BRASIL. Ministério da Educação. Nota técnica no 24 de 21 de março de 2013.

Orientação aos Sistemas de Ensino para a implementação da Lei no 12.764/2012, março 2013. Disponível em:

http://portal.mec.gov.br/index.php?option=com_docman\&view=download\&alias=13287nt24-sistem-lei12764-2012\&category_slug=junho-2013-pdf\&Itemid=30192. Acesso em: 23 set. 2016.

BRASIL. Ministério da Educação. Institui as Diretrizes Curriculares Nacionais para os cursos de graduação em Psicologia, estabelecendo normas para o projeto pedagógico complementar para a Formação de Professores de Psicologia. Resolução no 5, de 15 de março de 2011. Diário Oficial da União, Brasília, seção 1, 19-20, 2011.

BRASIL. Ministério da Educação. Resolução № 4, de 2 de outubro de 2009. Institui Diretrizes Operacionais para o Atendimento Educacional Especializado na Educação Básica, modalidade Educação Especial. Disponível em:

http://portal.mec.gov.br/dmdocuments/rceb004_09.pdf. Acesso em: 10 de setembro 2019.

CARMONA, Daniele Souza ; BATISTA, Cássia Beatriz. Cenários de Aprendizagem e Noções de Saúde: a percepção dos Estudantes de Psicologia sobre sua formação.

Psicologia em Revista (Online), v. 22, p. 46-68, 2016.

CECCIM, Ricardo Burg; CARVALHO, Yara Maria de. Ensino da saúde como projeto da integralidade: a educação dos profissionais de saúde no SUS. In: CECCIM, Ricardo Burg; Pinheiro, Roseni; Mattos, Ruben Araújo de (orgs.). Ensinar Saúde: a integralidade e o SUS nos cursos de graduação na área da saúde. Rio de Janeiro: IMS/UERJ, 2006. 
CELLARD, André. Análise documental. In: Poupart, Jean. et.al. A Pesquisa Qualitativa: enfoques epistemológicos e metodológicos. Trad.A. C. Nasser. Petrópolis: Vozes, 2008. p.295-316.

CIDAV, Zuleyha. et al. Cost offset associated with Early Start Denver Model for children with autism. Journal of the American Academy of Child \& Adolescent Psychiatry, v.56, n.9, 777-783, 2017. Disponível em: https://www.ncbi.nlm.nih.gov/pubmed/28838582. Acesso em: 4 mar.2018

COSTA, Daniela C. F. da. Intervenção Precoce no Transtorno do Espetro do Autismo. Dissertação. Lisboa: [s.n.], 2014. Disponível em:

https://comum.rcaap.pt/bitstream/10400.26/14422/1/DanielaCosta.pdf. Acesso em: 6 mai.2019.

DAWSON, Geraldine. Early behavioral intervention, brain plasticity, and the prevention of autism spectrum disorder. Developmentand Psychopatholy, 20(3), 775-803, 2008. doi: $10.1017 / \mathrm{s} 0954579408000370$

DSM-V - Manual de Diagnóstico e Estatística das Perturbações Mentais 4.ed. American Psychiatric Association, 2013.

FEUERWERKER, Laura; ALMEIDA, Márcio. Diretrizes Curriculares e Projetos Pedagógicos: é tempo de ação! Revista Brasileira de Enfermagem, v.56, n.4, 351-352, 2003.

GOMES, Camila Graciella Santos; MENDES, Enicéia Gonçalves. Escolarização inclusiva de alunos com autismo na rede municipal de ensino de Belo Horizonte. Revista Brasileira de Educação Especial, v.16, n.3, 375-396, 2010.

GRINKER, Roy; DALEY, Tamara; MANDELL, David. Culture and Autism. In: VOLKMAR, Fred (ed.). Encyclopedia of Autism Spectrum Disorders. New York: Springer, 2013.

KUHN, Roland. Eugen Bleuler's concepts of psychopathology. Hist. Psychiatry, v.15, n.3, 361-366, 2004.

INEP - Instituto Nacional de Estudos e Pesquisas Educacionais Anísio Teixeira. Ministério da Educação. (s/d). Senso da Educação Superior. Disponível em:

http://portal.inep.gov.br/educacao-superior. Acesso em: 02 jun. 2017.

KLIN, Ami. Autismo e síndrome de Asperger: uma visão geral. Revista Brasileira de Psiquiatria, n.28 (Suppl.1), 2006. Disponível em:

http://www.scielo.br/scielo.php?script=sci_arttext\&pid=S1516-44462006000500002.

Acesso em: 02 set. 2015.

NOGUEIRA, Maria Luísa Magalhães, VICARI, Luiza Leão; BRUN, Olívia Ameno.

Transtornos do espectro autista e inclusão na rede pública de Belo Horizonte: percepção dos familiares. In: CONGRESSO BRASILEIRO DE EDUCAÇÃO ESPECIAL, 7, 2016, São Carlos/SP. Anais do Sétimo Congresso Brasileiro de Educação Especial. São CarlosSP: UFSCar, 2016. 
NUNES, Debora Regina de Paula; AZEVEDO, Mariana Queiroz Orrico; SCHMIDT, Carlo. Inclusão educacional de pessoas com autismo no Brasil: uma revisão da literatura.

Revista Educação Especial, 26(47), 557-572, 2013.

ORTEGA, Francisco et al. A construção do diagnóstico do autismo em uma rede social virtual brasileira. Interface (Botucatu), v.17, n.44, 119-132, 2013. Disponível em: http://www.scielo.br/scielo.php?script=sci_arttext\&pid=S1414$32832013000100010 \&$ Ing=en\&nrm=isso. Acesso em: 10 maio 2015.

PACHECO, J. A. Notas para uma síntese de uma década de consolidação dos estudos curriculares. Investigar em Educação, São Paulo, v. 1, n. 1, p. 227-273, 2002.

PAULA, Cristiane Silvestre; BELISÁSIO FILHO, José Ferreira; TEIXEIRA, Maria Cristina Triguero Veloz. Estudantes de psicologia concluem a graduação com uma boa formação em autismo? Psicologia: Teoria e Prática, v.18, n.1, 20-221, 2016. Disponível em: http://pepsic.bvsalud.org/pdf/ptp/v18n1/16.pdf. Acesso em: 15 maio 2015.

PINTO, Rayssa N.M. et al. Autismo infantil: impacto do diagnóstico e repercussões nas relações familiares. Revista Gaúcha de Enfermagem, v.37, n.3, 2016.

RIBEIRO, Sabrina H. et al. Barriers to early identification of autism in Brazil. Rev. Bras. Psiquiatr., São Paulo, v.39, n.4, p. 352-354, dec. 2017. Disponível em: http://www.scielo.br/scielo.php?script=sci_arttext\&pid=S1516$44462017000400352 \&$ Ing=en\&nrm=iso. Acesso em: 5 jan. 2020.

ROGERS, Sally; VISMARA, Laurie. Evidence-based comprehensive treatments for early autism. Journal of Clinical Child and Adolescent Psychology, v.37, n.1, 8-38, 2008. doi: $10.1080 / 15374410701817808$

ROGERS, Sally; DAWSON, Geraldine. Intervenção Precoce em Crianças com Autismo. Lisboa: Lidel, 2014.

SILVA, Tomaz Tadeu da. Documentos de Identidade: uma introdução às teorias do currículo. 2 ed. Belo Horizonte: Autêntica, 2003.

SHAH, Kalpna. What do medical students know about autism? Autism, v.5, n.2, 127-133, 2001. https://doi.org/10.1177/1362361301005002003

WING, Lorna. The history of ideas on autism: legends, myths and reality. Autism, v.1, n.1, 13-23, 1997.Disponível em:

https://compass-training.weebly.com/uploads/1/3/9/6/13963732/wing_1997.pdf. Acesso em: 10 maio 2015. 
http://dx.doi.org/10.5902/1984686X40092

\section{Notas}

${ }^{1}$ A primeira vez que Bleuler utiliza o termo 'autismo' é num artigo de 1910 - Zur Theorie des schizophrenen Negativismus. Psychiatrisch-Neurologische Wochenschrift - que trata do negativismo esquizofrênico: "What I understand as 'autism' is about the same as Freud's 'autoerotism', but I prefer to avoid using this expression, because everybody who does not know Freud's writings exactly misunderstands it." (Bleuler apud Kuhn, 2004, p.364)

2 "The understanding of autism can only be gained by further examination both across and within cultures." [tradução nossa]

${ }^{3}$ As Matrizes Curriculares foram os documentos de acesso mais fácil que constam nos sites institucionais. Estes documentos apresentam os nomes das disciplinas por período e às vezes são nomeadas como Grade Curricular.

${ }^{4}$ Os Planos de Ensino são documentos presentes nos Projetos pedagógicos dos cursos, entretanto, podem sofrer algumas alterações a cada edição da disciplina de acordo com o professor, por isso buscamos coletar Planos e Ensino atuais. Uma das instituições só disponibilizaria o acesso a esses documentos mediante pagamento de taxa, o que inviabilizou sua participação no estudo

\section{Correspondência}

Jardel Sander da Silva - Universidade Federal de Minas Gerais, Faculdade de Educação, Departamento de Métodos e Técnicas de Ensino, Pampulha, Belo Horizonte, Minas Gerais, Brasil.

CEP: 31270-901

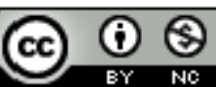

This work is licensed under a Creative Commons Attribution-NonCommercial 4.0 International (CC BY-NC 4.0) 